\title{
Impact of low-energy nuclear excitations on neutrino-nucleus scattering at MiniBooNE and T2K kinematics
}

\author{
V. Pandey, ${ }^{1, *}$ N. Jachowicz, ${ }^{1}$ M. Martini, ${ }^{1,2}$ R. González-Jiménez, ${ }^{1}$ J. Ryckebusch, ${ }^{1}$ T. Van Cuyck, ${ }^{1}$ and N. Van Dessel ${ }^{1}$ \\ ${ }^{1}$ Department of Physics and Astronomy, Ghent University, Proeftuinstraat 86, B-9000 Gent, Belgium \\ ${ }^{2}$ ESNT, CEA, IRFU, Service de Physique Nucléaire, Université de Paris-Saclay, F-91191 Gif-sur-Yvette Cedex, France
}

(Received 5 July 2016; revised manuscript received 27 September 2016; published 7 November 2016)

\begin{abstract}
Background: Meticulous modeling of neutrino-nucleus interactions is essential to achieve the unprecedented precision goals of present and future accelerator-based neutrino-oscillation experiments.

Purpose: Confront our calculations of charged-current quasielastic cross sections with the measurements of MiniBooNE and T2K, and to quantitatively investigate the role of nuclear-structure effects, in particular, lowenergy nuclear excitations in forward muon scattering.

Method: The model takes the mean-field approach as the starting point, and solves Hartree-Fock (HF) equations using a Skyrme (SkE2) nucleon-nucleon interaction. Long-range nuclear correlations are taken into account by means of the continuum random-phase approximation (CRPA) framework.

Results: We present our calculations on flux-folded double differential, and flux-unfolded total cross sections off ${ }^{12} \mathrm{C}$ and compare them with MiniBooNE and (off-axis) T2K measurements. We discuss the importance of low-energy nuclear excitations for the forward bins.

Conclusions: The HF and CRPA predictions describe the gross features of the measured cross sections. They underpredict the data (more in the neutrino than in the antineutrino case) because of the absence of processes beyond pure quasielastic scattering in our model. At very forward muon scattering, low-energy HF-CRPA nuclear excitations $(\omega<50 \mathrm{MeV})$ account for nearly $50 \%$ of the flux-folded cross section. This extra low-energy strength is a feature of the detailed microscopic nuclear model used here, that is not accessed in a Fermi-gas based approach.
\end{abstract}

DOI: 10.1103/PhysRevC.94.054609

\section{INTRODUCTION}

The study of neutrino oscillations is moving into an era of precision with an intense enhancement in the activities of accelerator-based neutrino-oscillation experiments. Substantial progress has been made in the determination of the mass-squared differences and mixing angles. However, in order to improve the precision of the analysis, a rigorous description of neutrino-nucleus cross sections is required. The progress and issues related to the cross sections in this context were recently reviewed in Refs. [1-3]. In recent years, several collaborations have reported muon neutrino cross sections on nuclei [4-25]. The challenges faced in these efforts, and especially those related to the neutrino-nucleus signal in the detector, need detailed microscopic neutrino-interaction models that can describe the variety of nuclear effects over the broad kinematical range probed. A thorough comparison of the cross-section measurements with theoretical predictions is crucial to assess the role of nuclear effects in the target's response and to reduce the systematic uncertainties in the extraction of the oscillation parameters.

In this work, we aim at discussing the results of calculations for charged-current (CC) $v_{\mu}$ and $\bar{v}_{\mu}$ scattering on ${ }^{12} \mathrm{C}$, at the kinematics of the MiniBooNE and $\mathrm{T} 2 \mathrm{~K}$ experiments. In particular, we focus on comparing our calculations with charged-current quasielastic (CCQE) $v_{\mu}$ and $\bar{v}_{\mu}$ measurements of MiniBooNE [4,11], and inclusive and CCQE $v_{\mu}$ measure-

*Vishvas.Pandey@UGent.be ments of (off-axis near detector ND280) T2K [12,24]. One of the major objectives of this work is the investigation of the role of neutrino-induced low-energy nuclear collective excitations in MiniBooNE and T2K's signal. To this end we adopt a continuum random-phase approximation (CRPA) model.

The article is organized as follows. In Sec. II, we briefly discuss the main ingredients of our model. Section III is divided in three parts: We compare the flux-folded doubledifferential CRPA cross sections with the measurements of MiniBooNE and T2K in Sec. III A. In order to asses the contributions stemming from low-energy nuclear excitations, we discuss the specific case of forward muon scattering bins in Sec. III B. In Sec. III C, we show flux-unfolded total cross sections. The conclusions are presented in Sec. IV.

\section{FORMALISM}

The CRPA model was originally developed to describe exclusive electron- and photo-induced nucleon knockout reactions [26,27]. The model was later used to predict neutrino scattering at supernova energies both in charged-current (CC) and neutral-current (NC) reactions [28-31]. The formalism was further extended to the $\mathrm{QE}$ reaction region and successfully tested against electron-scattering data for a variety of nuclear targets in the QE region [32-34]. Here, we briefly summarize the essence of our model. The starting point of the description of the nuclear dynamics is a mean field (MF). We solve the Hartree-Fock (HF) equations using the Skyrme SkE2 nucleon-nucleon interaction [27,35]. Once the bound and continuum single-nucleon wave functions are determined, 
long-range correlations are taken into account by means of a CRPA approach based on a Green's function formalism. The CRPA describes an excited state as a linear combination of particle-hole (1p1h) and hole-particle (1h1p) excitations out of a correlated nuclear ground state

$$
\left|\Psi_{R P A}^{C}\right\rangle=\sum_{C^{\prime}}\left[X_{C, C^{\prime}}\left|p^{\prime} h^{\prime-1}\right\rangle-Y_{C, C^{\prime}}\left|h^{\prime} p^{\prime-1}\right\rangle\right]
$$

where $C$ represents the complete set of quantum numbers of an accessible single-nucleon knockout channel. The RPA polarization propagator $\Pi^{(R P A)}$ is obtained by the iteration of the first order contributions to the particle-hole Green's function $\Pi^{(0)}$ and is obtained as the solution to the equation

$$
\begin{aligned}
& \Pi^{(R P A)}\left(x_{1}, x_{2} ; E_{x}\right) \\
& =\Pi^{(0)}\left(x_{1}, x_{2} ; E_{x}\right)+\frac{1}{\hbar} \int d x d x^{\prime} \Pi^{0}\left(x_{1}, x ; E_{x}\right) \\
& \quad \times \tilde{V}\left(x, x^{\prime}\right) \Pi^{(R P A)}\left(x^{\prime}, x_{2} ; E_{x}\right)
\end{aligned}
$$

where $E_{x}$ is the excitation energy of the target nucleus and $x$ is a shorthand notation for the combination of the spatial, spin, and isospin coordinates. The $\Pi^{(0)}$ in Eq. (2) corresponds to the HF contribution to the polarization propagator and $\tilde{V}$ denotes the antisymmetrized nucleon-nucleon SkE2 interaction.

The SkE2 interaction was optimized against ground-state and low-excitation properties of spherical nuclei. Its strength lies in its ability to describe nuclear excitations in the few tens of $\mathrm{MeV}$ energy range. The same SkE2 two-body interaction, that is used to solve the HF equations, is used to calculate the CRPA polarization propagator. In order to restrain the SkE2 force from becoming unrealistically strong at high virtuality $Q^{2}$, a dipole hadronic form factor is introduced at the nucleon-nucleon interaction vertices [33]. The continuum wave functions are obtained by solving the positive-energy Schrödinger equation with appropriate boundary conditions. Hence, the distortion effects (escape width) from the residual nucleons on the outgoing nucleon is taken into account. A folding procedure is used to take into account also the spreading width of the particle states [33], which makes the description of giant resonances more realistic within the CRPA approach. In order to consider the influence of the nuclear Coulomb field on the outgoing lepton, a modified effective momentum approximation [36] is used. Further, to improve our description at higher momentum transfers, we have implemented relativistic kinematic corrections [37]. The world-averaged axial mass value $M_{A}=1.03 \mathrm{GeV}$ was used for all the calculations in this paper.

\section{CROSS SECTION ANALYSIS}

Both MiniBooNE and T2K use a target rich in ${ }^{12} \mathrm{C}$. Their fluxes $[4,11,12]$ are slightly different, as shown in Fig. 1. Both $v_{\mu}$ beams have average energies around $800 \mathrm{MeV}$ while the $\bar{v}_{\mu}$ MiniBooNE beam has a slightly lower average energy. The T2K beam is more sharply peaked, and receives less contributions beyond $1 \mathrm{GeV}$, than the MiniBooNE one.

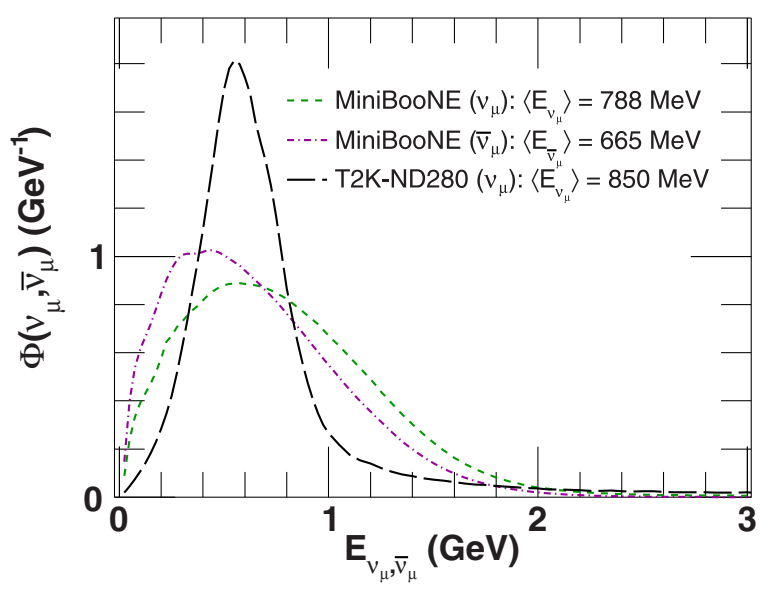

FIG. 1. Normalized MiniBooNE $v_{\mu}$ [4], $\bar{v}_{\mu}$ [11], and T2K [12] (off-axis ND280) $v_{\mu}$ fluxes.

\section{A. Flux-folded double differential cross sections}

We present CC pure QE neutrino cross sections folded with the MiniBooNE flux in Fig. 2. The top panels are plotted as a function of the muon scattering angle $\cos \theta_{\mu}$ for several bins of muon kinetic energies $T_{\mu}$ and the bottom panels are plotted as function of $T_{\mu}$ for different ranges of $\cos \theta_{\mu}$. The calculated cross sections are averaged over the $T_{\mu}$ and $\cos \theta_{\mu}$ ranges. We compare HF and CRPA calculations with the experimental data of MiniBooNE [4]. The HF and CRPA calculations reproduce the shape of the measured cross sections. In the top panels, the CRPA cross sections are slightly higher than the $\mathrm{HF}$ ones for $\cos \theta_{\mu}$ approaching 1, owing to extra contributions stemming from low-energy excitations. For forward scattering the $1 \mathrm{p}-1 \mathrm{~h}$ CRPA model reasonably reproduces the data, whereas it tends to underestimate the measured cross sections at backward scattering. The measurement of CCQE neutrino [4] and antineutrino [11] cross sections by the MiniBooNE collaboration sparked off discussions about the nuclear effects active in the broad energy range covered by the flux. The CCQE(-like) cross section in MiniBooNE is defined as the process where one muon and no pions are observed in the final state. Corrections to genuine QE processes stem from multinucleon correlations in the target nuclei. Those multinucleon processes [like meson-exchange currents (MEC), $\Delta$-isobar currents and short-range correlations] give rise to additional sources of strength in the nuclear response: a correction in the single-nucleon knockout channel, and a nonvanishing strength in multinucleon knockout channel. The necessity to include multinucleon effects to successfully describe the CCQE MiniBooNE data has been suggested [38,39] and confirmed by several independent models [40-50]. As expected, the exclusion of multinucleon channels in this work, results in an underestimation of the data.

In Fig. 3, we compare our flux-folded predictions for antineutrino cross sections with the MiniBooNE measurements of Ref. [11]. In this case, the CRPA predictions are closer to the MiniBooNE data than those for the neutrino calculations. This again confirms that the relative role of multinucleon excitations is more important for the neutrino than for the antineutrino cross sections, as discussed in Ref. [39]. 

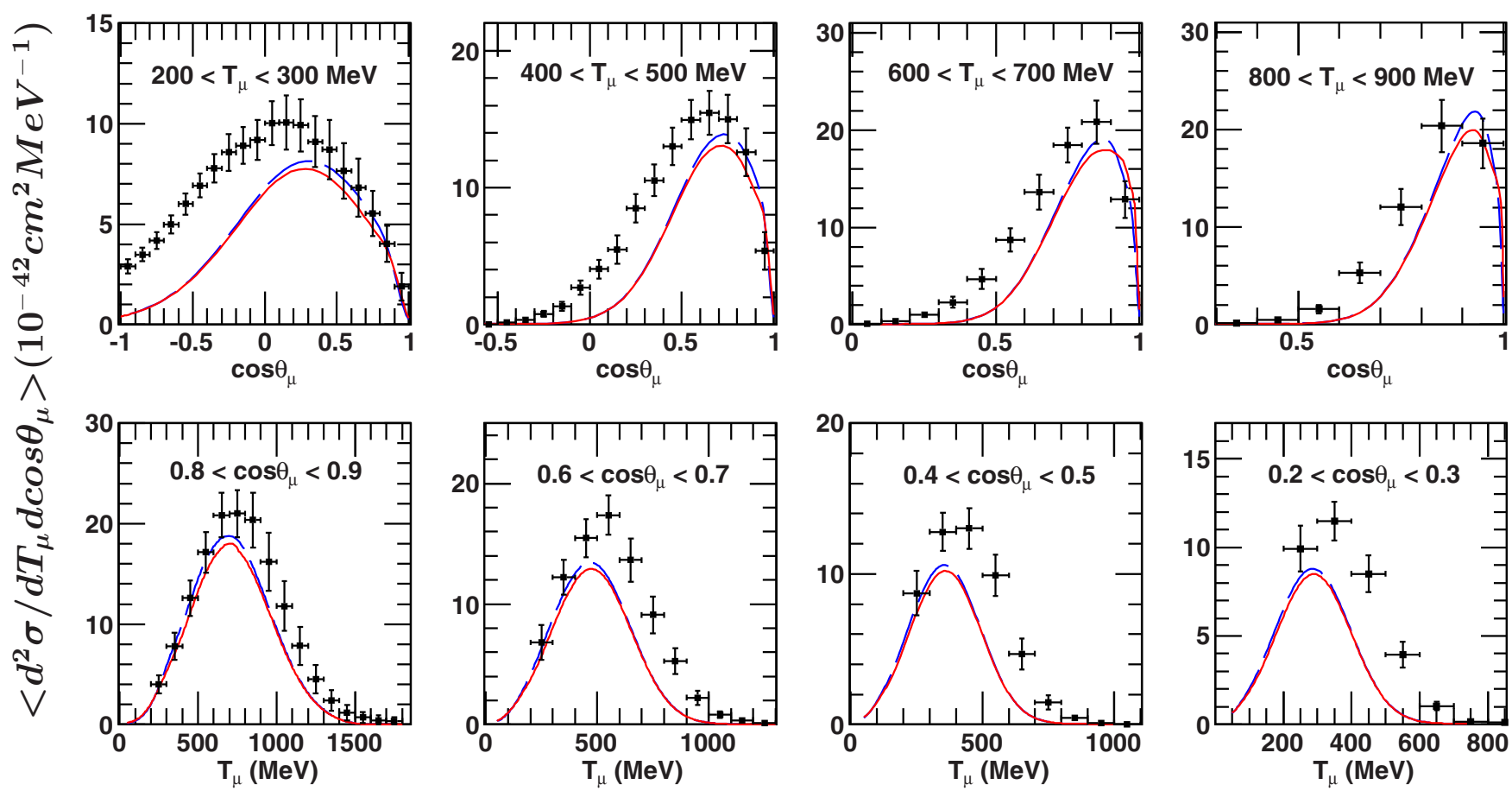

FIG. 2. MiniBooNE flux-folded double-differential cross sections per target neutron for ${ }^{12} \mathrm{C}\left(v_{\mu}, \mu^{-}\right) X$, plotted as a function of cos $\theta_{\mu}$ for different $T_{\mu}$ values (top) and as a function of $T_{\mu}$ for different ranges of $\cos \theta_{\mu}$ (bottom). Solid curves are CRPA and dashed curves are HF results. MiniBooNE data including shape uncertainties are taken from Ref. [4]. The data contain an additional normalization uncertainty of $10.7 \%$, not included here.
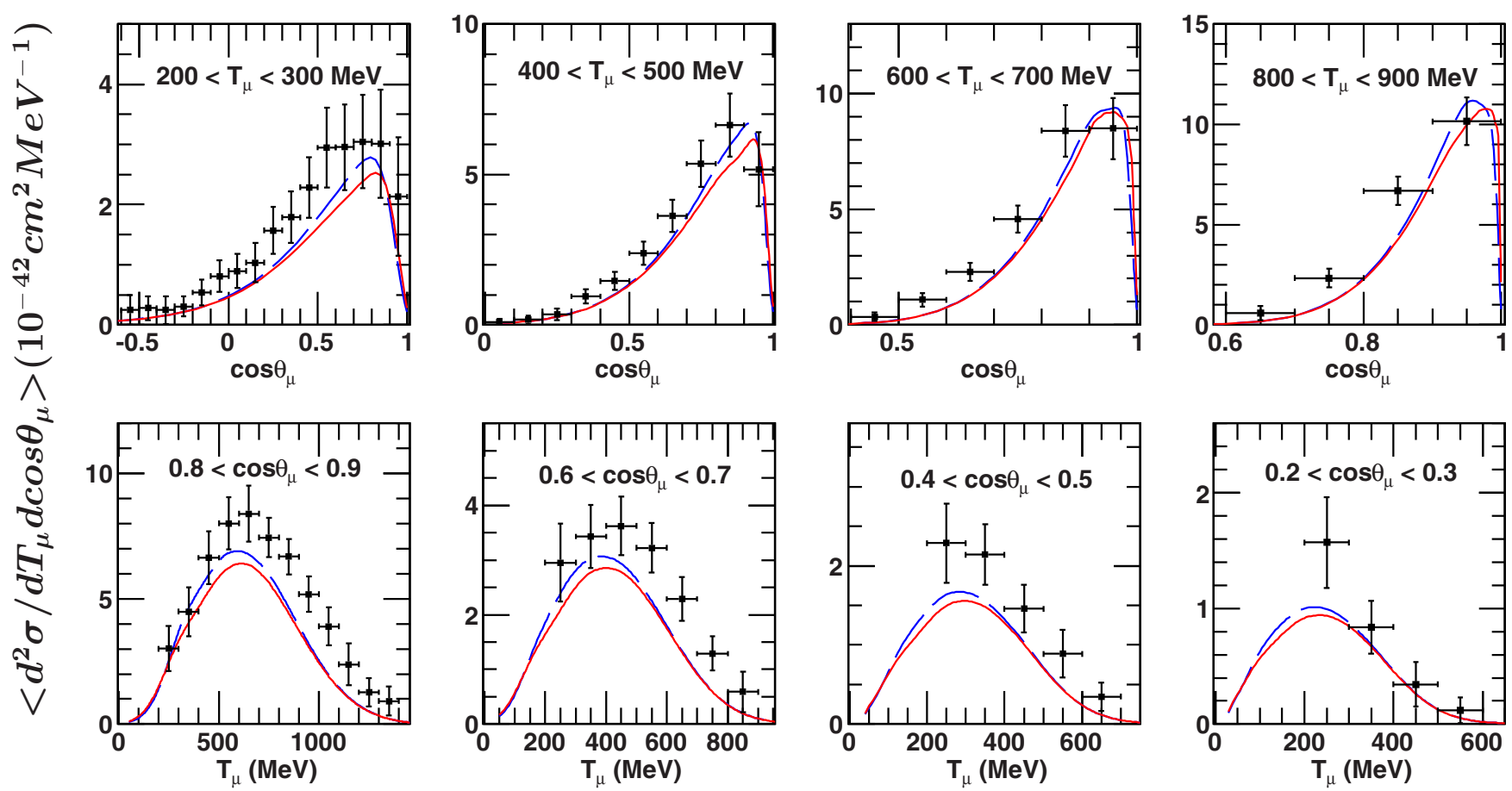

FIG. 3. Same as Fig. 2 but for the process ${ }^{12} \mathrm{C}\left(\bar{v}_{\mu}, \mu^{+}\right) X$. Solid curves are CRPA and dashed curves are HF calculations. MiniBooNE data including shape uncertainties are taken from Ref. [11]. The data contain an additional normalization uncertainty of $17.4 \%$, not included here. 


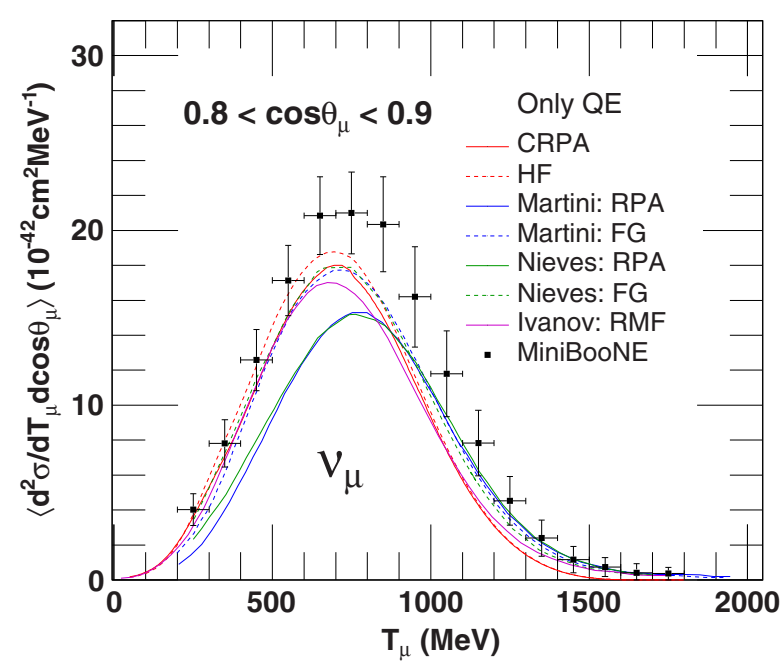

FIG. 4. MiniBooNE flux-folded cross section per target neutron for ${ }^{12} \mathrm{C}\left(v_{\mu}, \mu^{-}\right) X$ at $0.8<\cos \theta_{\mu}<0.9$. The CRPA and HF predictions are compared with those of Martini et al. [43], Nieves et al. [44], and Ivanov et al. [51].

In Fig. 4, we present a QE-only comparison of our HF and CRPA calculations with the predictions of Martini et al. [43], Nieves et al. [44], and Ivanov et al. [51]. The comparison is presented for MiniBooNE flux-folded cross sections off ${ }^{12} \mathrm{C}$ at $0.8<\cos \theta_{\mu}<0.9$. In the Martini et al. and Nieves et al. approaches, the $\mathrm{QE}$ predictions of both models (FG and RPA) almost coincide. There is a sizable RPA quenching in the predictions of both Martini et al. and Nieves et al. The size of the quenching is smaller in the CRPA, resulting in a larger predicted cross section for the $\mathrm{QE}$ process. The authors of Refs. $[43,44]$ attribute the strong quenching in their model to the explicit inclusion of the Ericson-Ericson-LorentzLorentz effect, which accounts for the possibility of a $\Delta$-hole excitation in the RPA chain. For a more detailed comparison of our HF-CRPA model with the model of Martini et al., we refer the reader to Ref. [34]. The relativistic mean-field (RMF) predictions of Ivanov et al. are lower than our HF ones around the peak, but the RMF generates more strength at the high- $T_{\mu}$ end. The pure QE RPA results of Martini et al. and Nieves $e t a l$. are significantly different from HF, CRPA, and RMF results. These difference can be assigned to the use of a detailed microscopic nuclear model in the HF and RMF calculations compared to the FG ones. Note that the additional contribution from $n \mathrm{p}-n \mathrm{~h}$ in Martini $e t a l$. and Nieves et al., and from meson-exchange current (MEC) in Ref. [52] were included in these collaborations to describe the MiniBooNE data. These additional channels are not shown in Fig. 4 as we focused only on the pure QE channel. Still, one should be aware that the separation into different channels can be strongly model-dependent.

The T2K collaboration reported on CC-inclusive doubledifferential cross sections as a function of muon momentum $p_{\mu}$ and scattering angle $\cos \theta_{\mu}$ [12], and CCQE total cross sections as a function of incident neutrino energies [24]. Reference [53] finds a satisfactory agreement with the T2K data, after
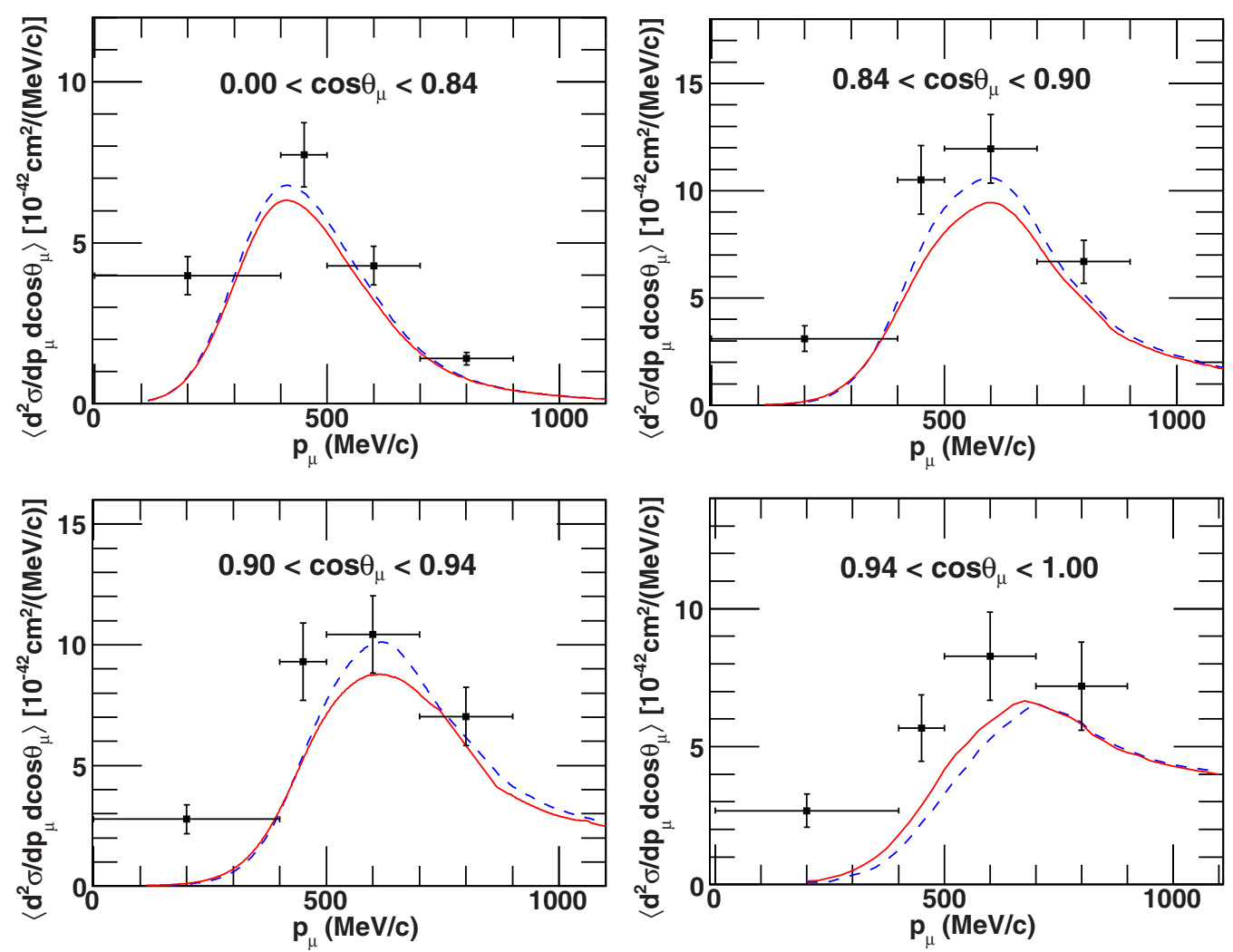

FIG. 5. T2K flux-folded inclusive $\mathrm{CC}$ double-differential cross sections per target nucleon on ${ }^{12} \mathrm{C}$ plotted as a function of muon momentum $p_{\mu}$, for different bins of $\cos \theta_{\mu}$. CRPA (solid curves) and HF (dashed-curves) are compared with T2K measurements of [12]. 
inclusion of multinucleon and single-pion production channels. On the other hand, the relativistic Green's function (RGF) approach of Ref. [54], which successfully describes the MiniBooNE data, underestimates the T2K results. Another comparison is presented in the superscaling approach of Ref. [55].

We have computed the T2K $v_{\mu}$ flux-folded QE doubledifferential cross sections. Our HF and CRPA results are confronted with the data in Fig. 5. The cross sections are averaged over each $\cos \theta_{\mu}$ bin. CRPA cross sections reproduce the gross feature of the T2K data, but underestimate the data, as can be expected in absence of effects beyond QE. The underestimation is more pronounced for smaller values of $p_{\mu}$, which corresponds to the higher excitation energies where the inelastic channels beyond QE can be expected to have substantial contributions. For the most forward bin $\left(0.94<\cos \theta_{\mu}<1.0\right)$, the CRPA cross section is higher than the $\mathrm{HF}$ one for $p_{\mu} \lesssim 700 \mathrm{MeV} / c$. This behavior can be attributed to giant-resonances contributing a portion of the CRPA strength.

\section{B. Forward scattering cross section}

In Ref. [33], we stressed the importance of low-energy nuclear excitations for the forward muon scattering events in MiniBooNE and T2K. Here we compare the most forward bin of the MiniBooNE and T2K data sets to explore the contributions emerging from low-energy excitations in these experiments. A substantial amount of the cross section strength in this kinematic region, where the excitation energy of the nucleus is $\lesssim 50 \mathrm{MeV}$, arises from collective nuclear excitations. As we have shown in Fig. 14 of Ref. [33] and Fig. 9 of Ref. [34], at these kinematics the longitudinal response generates major strength or comparable strength (depending on the neutrino energy) of the cross section with respect to the transverse response. Models that do not include collective effects can be

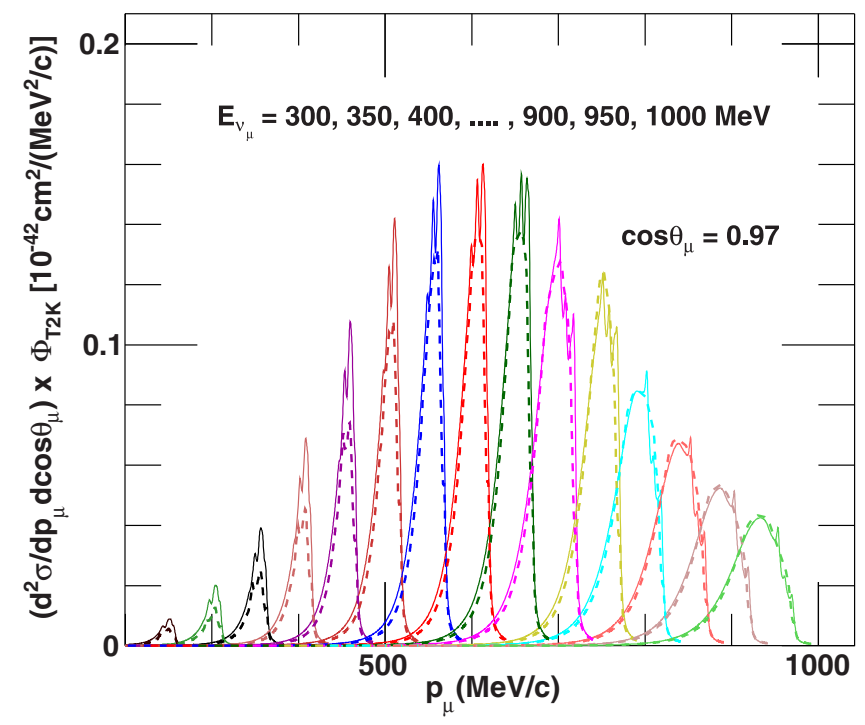

FIG. 6. Double differential CRPA (solid curves) and HF (dotted curves) cross sections on ${ }^{12} \mathrm{C}$ for $\cos \theta_{\mu}=0.97$ and for fixed neutrino energies from $300 \mathrm{MeV}$ to $1000 \mathrm{MeV}$, weighted with the T2K $v_{\mu}$ flux (of Fig. 1) and plotted as a function of $p_{\mu}$.

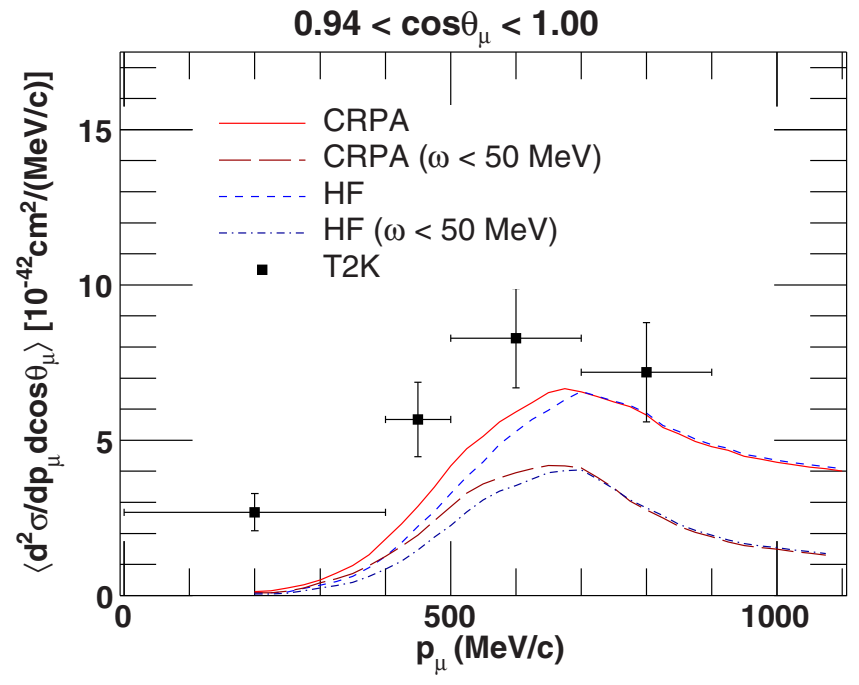

FIG. 7. The most forward bin, $0.94<\cos \theta_{\mu}<1.0$, of Fig. 5 . $\mathrm{T} 2 \mathrm{~K}$ data are taken from Ref. [12].

expected to underestimate the data at small scattering angles. The RGF predictions for T2K [54] significantly underestimate the data for $0.94<\cos \theta_{\mu}<1$. In Ref. [53], even after the inclusion of multinucleon and one-pion production channels,

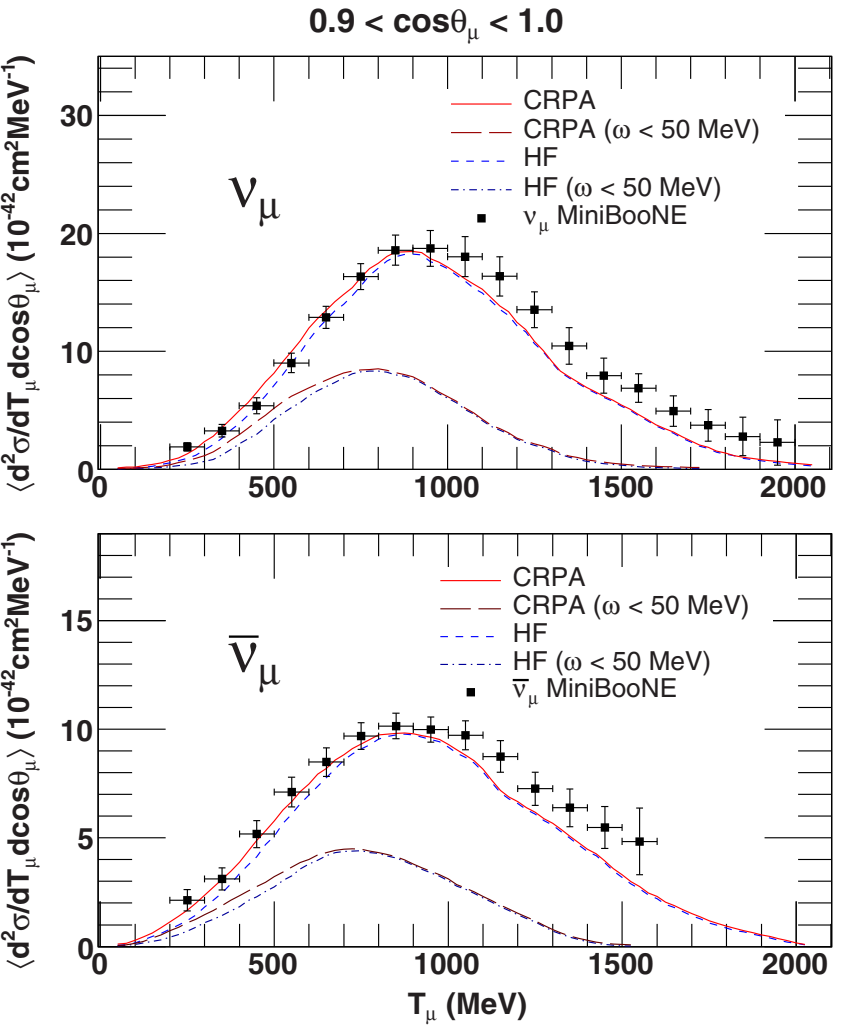

FIG. 8. The $T_{\mu}$ dependence of the CCQE double-differential cross sections per target neutron folded with MiniBooNE flux, for $0.9<\cos \theta_{\mu}<1.0$. CRPA calculations are compared with MiniBooNE data of Ref. [4]. Experimental error bars represent the shape uncertainties. 

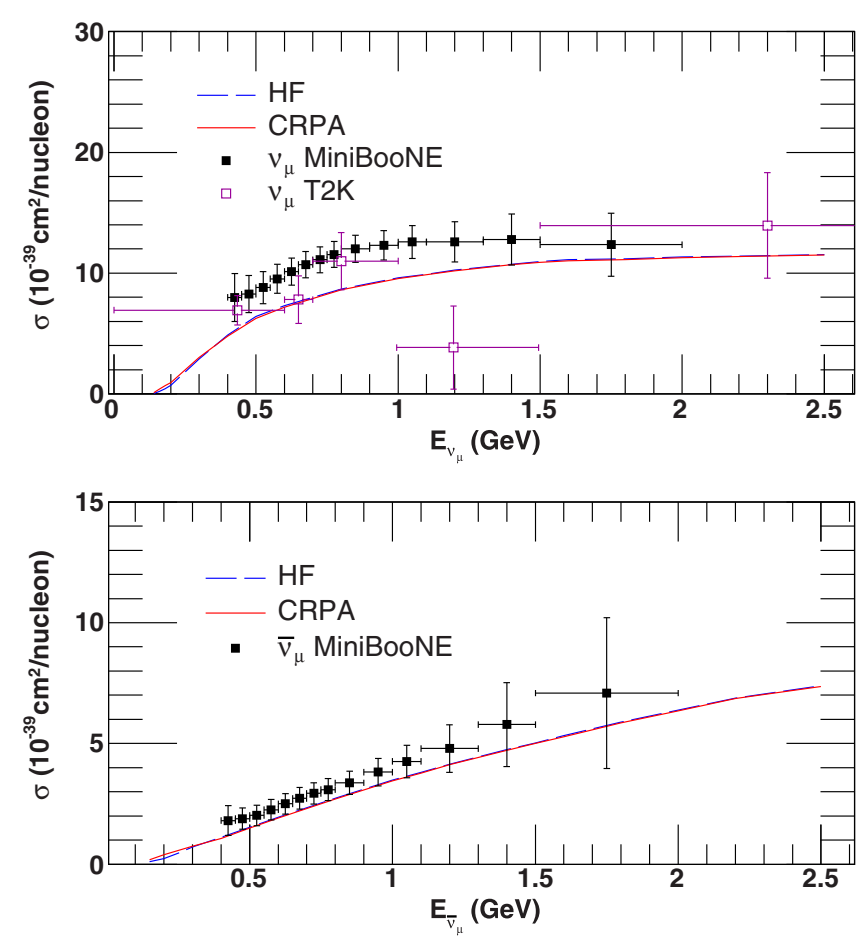

FIG. 9. Total $\mathrm{CCQE}{ }^{12} \mathrm{C}\left(v_{\mu}, \mu^{-}\right) X$ and ${ }^{12} \mathrm{C}\left(\bar{v}_{\mu}, \mu^{+}\right) X$ cross sections per target nucleon plotted as a function of (anti)neutrino energy. The experimental data are taken from MiniBooNE $\left(v_{\mu}\right)$ [4], T2K $\left(v_{\mu}\right)[24]$, and MiniBooNE $\left(\bar{v}_{\mu}\right)[11]$.

which reproduced the data successfully in other angular bins, the prediction lacks strength in the forward bin. In Fig. 6, we show HF and CRPA cross sections weighted with the T2K flux, for fixed neutrino energies from $300 \mathrm{MeV}$ to 1000 $\mathrm{MeV}$ and for fixed scattering angle $\cos \theta_{\mu}=0.97$. At these kinematics, the low-energy excitations significantly contribute to the cross section. In fact, the contributions from $\omega<50$ $\mathrm{MeV}$ processes constitute a large part of the cross section for most incoming neutrino energies contributing to this forward angular bin. As expected, the low-energy excitation peaks are more pronounced in CRPA calculation than in HF ones even up to incoming energies of $E_{v}=1000 \mathrm{MeV}$. Though, the total integrated strength of both HF and CRPA are almost same for $E_{v} \gtrsim 700 \mathrm{MeV}$. Flux-folding of these cross sections washes out the low-energy excitation peaks and smooths the overall curve. Still, the important low energy strength remains. In Fig. 7, we show the contribution emerging from low-energy excitations $(\omega<50 \mathrm{MeV})$ for the most forward bin of Fig. 5 . This strength accounts for nearly 50\% of the cross section in this kinematic bin, representing lepton scattering angles up to $20^{\circ}$. The relativistic Fermi-gas (RFG) based models, implemented in Monte Carlo generators, in principle are not suitable to provide a detailed description of this kinematic range. For the sake of completeness, in Fig. 8 we compare our flux-folded results for $0.90<\cos \theta_{\mu}<1$ with the MiniBooNE measurements and separately plot the contribution from $\omega<50 \mathrm{MeV}$. This hints at the importance of an accurate description of neutrino-induced low-energy nuclear excitations in the most forward MiniBooNE and T2K measurements.

\section{Total cross section}

In Fig. 9, we compare the computed CCQE ${ }^{12} \mathrm{C}\left(v_{\mu}, \mu^{-}\right) X$ and ${ }^{12} \mathrm{C}\left(\bar{v}_{\mu}, \mu^{+}\right) X$ total cross section with the data of MiniBooNE [4,11] and T2K [24]. Unlike the double-differential ones, the total experimental cross sections are model dependent as they are expressed as a function of reconstructed energy [56-60], while the theoretical results as a function of true energy. On average, the strength of the MiniBooNE measurements is higher than the T2K 'QE-like' one. The measurements of these two data sets are quite comparable except for $E_{v_{\mu}} \simeq$ $1.2 \mathrm{GeV}$. The CRPA calculations are within the error bar of the T2K data, but underpredict the MiniBooNE ones. The CRPA results agree much better with the antineutrino measurement of MiniBooNE. The HF and CRPA cross sections in both the neutrino and antineutrino case are almost coinciding with each other except for $E<250 \mathrm{MeV}$ where CRPA cross section is higher than the HF one.

\section{CONCLUSIONS}

We have calculated $v_{\mu^{-}}{ }^{12} \mathrm{C}$ and $\bar{v}_{\mu^{-}}{ }^{12} \mathrm{C}$ cross sections in kinematics corresponding with the MiniBooNE and T2K experiments. We compared flux-folded double differential cross sections with CCQE $v_{\mu}$ and $\bar{v}_{\mu}$ MiniBooNE measurements, and with inclusive T2K (off-axis) measurements. The CRPA cross sections compare favorably to the shape but underestimate the MiniBooNE data for backward muon scattering angles. The missing strength can be associated with the contribution from multinucleon knockout and single-pion production processes. Still, a comparison of the flux-folded cross sections of MiniBooNE and T2K, shows that for forward muon scattering, the neutrino-induced low-energy nuclear excitations $(\omega<50 \mathrm{MeV})$ account for nearly $50 \%$ of the flux-folded cross section. These contributions, inaccessible in RFG-based Monte Carlo generators, make a strong case for a more careful modeling of the forward signal in MiniBooNE and $\mathrm{T} 2 \mathrm{~K}$-like experiments.

\section{ACKNOWLEDGMENTS}

This work was supported by the Interuniversity Attraction Poles Programme initiated by the Belgian Science Policy Office (BriX network P7/12) and the Research Foundation Flanders (FWO-Flanders). M.M. acknowledges also the support and the framework of the "Espace de Structure et de réactions Nucléaire Théorique" (ESNT, http://esnt.cea.fr) at CEA.
[1] J. G. Morfin, J. Nieves, and J. T. Sobczyk, Adv. High Energy Phys. 2012, 934597 (2012).
[2] L. Alvarez-Ruso, Y. Hayato, and J. Nieves, New J. Phys. 16, 075015 (2014). 
[3] U. Mosel, arXiv:1602.00696 [nucl-th].

[4] A. A. Aguilar-Arevalo et al. (MiniBooNE Collaboration), Phys. Rev. D 81, 092005 (2010).

[5] A. A. Aguilar-Arevalo et al. (MiniBooNE Collaboration), Phys. Rev. D 81, 013005 (2010).

[6] A. A. Aguilar-Arevalo et al. (MiniBooNE Collaboration), Phys. Rev. D 82, 092005 (2010).

[7] Y. Nakajima et al. (SciBooNE Collaboration), Phys. Rev. D 83, 012005 (2011).

[8] A. A. Aguilar-Arevalo et al. (MiniBooNE Collaboration), Phys. Rev. D 83, 052007 (2011).

[9] A. A. Aguilar-Arevalo et al. (MiniBooNE Collaboration), Phys. Rev. D 83, 052009 (2011).

[10] C. Anderson et al. (ArgoNeuT Collaboration), Phys. Rev. Lett. 108, 161802 (2012)

[11] A. A. Aguilar-Arevalo et al. (MiniBooNE Collaboration), Phys. Rev. D 88, 032001 (2013).

[12] K. Abe et al. (T2K Collaboration), Phys. Rev. D 87, 092003 (2013).

[13] G. A. Fiorentini et al. (MINERvA Collaboration), Phys. Rev. Lett. 111, 022502 (2013).

[14] L. Fields et al. (MINERvA Collaboration), Phys. Rev. Lett. 111, 022501 (2013).

[15] R. Acciarri et al. (ArgoNeuT Collaboration), Phys. Rev. D 89, 112003 (2014).

[16] K. Abe et al. (T2K Collaboration), Phys. Rev. D 90, 052010 (2014).

[17] A. Higuera et al. (MINERvA Collaboration), Phys. Rev. Lett. 113, 261802 (2014).

[18] B. Eberly et al. (MINERvA Collaboration), Phys. Rev. D 92, 092008 (2015).

[19] R. Acciarri et al. (ArgoNeuT Collaboration), Phys. Rev. Lett. 113, 261801 (2014).

[20] T. Walton et al. (MINERvA Collaboration), Phys. Rev. D 91, 071301 (2015).

[21] T. Le et al. (MINERvA Collaboration), Phys. Lett. B 749, 130 (2015).

[22] A. A. Aguilar-Arevalo et al. (MiniBooNE Collaboration), Phys. Rev. D 91, 012004 (2015).

[23] K. Abe et al. (T2K Collaboration), Phys. Rev. D 91, 112002 (2015).

[24] K. Abe et al. (T2K Collaboration), Phys. Rev. D 92, 112003 (2015).

[25] K. Abe et al. (T2K Collaboration), Phys. Rev. D 93, 112012 (2016).

[26] J. Ryckebusch, M. Waroquier, K. Heyde, J. Moreau, and D. Ryckbosch, Nucl. Phys. A 476, 237 (1988).

[27] J. Ryckebusch, K. Heyde, D. Van Neck, and M. Waroquier, Nucl. Phys. A 503, 694 (1989).

[28] N. Jachowicz, S. Rombouts, K. Heyde, and J. Ryckebusch, Phys. Rev. C 59, 3246 (1999).

[29] N. Jachowicz, K. Heyde, J. Ryckebusch, and S. Rombouts, Phys. Rev. C 65, 025501 (2002).

[30] N. Jachowicz, K. Vantournhout, J. Ryckebusch, and K. Heyde, Phys. Rev. Lett. 93, 082501 (2004).

[31] N. Jachowicz and G. C. McLaughlin, Phys. Rev. Lett. 96, 172301 (2006).

[32] V. Pandey, N. Jachowicz, J. Ryckebusch, T. Van Cuyck, and W. Cosyn, Phys. Rev. C 89, 024601 (2014).
[33] V. Pandey, N. Jachowicz, T. Van Cuyck, J. Ryckebusch, and M. Martini, Phys. Rev. C 92, 024606 (2015).

[34] M. Martini, N. Jachowicz, M. Ericson, V. Pandey, T. Van Cuyck, and N. Van Dessel, Phys. Rev. C 94, 015501 (2016).

[35] M. Waroquier, J. Ryckebusch, J. Moreau, K. Heyde, N. Blasi, S. Y. van de Werf, and G. Wenes, Phys. Rep. 148, 249 (1987).

[36] J. Engel, Phys. Rev. C 57, 2004 (1998).

[37] S. Jeschonnek and T. W. Donnelly, Phys. Rev. C 57, 2438 (1998).

[38] M. Martini, M. Ericson, G. Chanfray, and J. Marteau, Phys. Rev. C 80, 065501 (2009).

[39] M. Martini, M. Ericson, G. Chanfray, and J. Marteau, Phys. Rev. C 81, 045502 (2010).

[40] J. E. Amaro et al., Phys. Lett. B 696, 151 (2011).

[41] J. Nieves, I. R. Simo, and M. J. Vicente Vacas, Phys. Rev. C 83, 045501 (2011).

[42] A. Bodek, H. Budd, and M. E. Christy, Eur. Phys. J. C 71, 1726 (2011).

[43] M. Martini, M. Ericson, and G. Chanfray, Phys. Rev. C 84, 055502 (2011).

[44] J. Nieves, I. Ruiz Simo, and M. J. Vicente Vacas, Phys. Lett. B 707, 72 (2012).

[45] J. E. Amaro, M. B. Barbaro, J. A. Caballero, and T. W. Donnelly, Phys. Rev. Lett. 108, 152501 (2012).

[46] O. Lalakulich, K. Gallmeister, and U. Mosel, Phys. Rev. C 86, 014614 (2012).

[47] J. Nieves, I. Ruiz Simo, and M. J. Vicente Vacas, Phys. Lett. B 721, 90 (2013).

[48] M. Martini and M. Ericson, Phys. Rev. C 87, 065501 (2013).

[49] G. D. Megias, T. W. Donnelly, O. Moreno, C. F. Williamson, J. A. Caballero, R. Gonzalez-Jimenez, A. De Pace, M. B. Barbaro, W. M. Alberico, M. Nardi, and J. E. Amaro, Phys. Rev. D 91, 073004 (2015).

[50] T. Van Cuyck, N. Jachowicz, R. González-Jiménez, M. Martini, V. Pandey, J. Ryckebusch, and N. Van Dessel, Phys. Rev. C 94, 024611 (2016).

[51] M. V. Ivanov, R. González-Jiménez, J. A. Caballero, M. B. Barbaro, T. W. Donnelly, and J. M. Udias, Phys. Lett. B 727, 265 (2013).

[52] G. D. Megias, J. E. Amaro, M. B. Barbaro, J. A. Caballero, T. W. Donnelly, and I. Ruiz Simo, arXiv:1607.08565 [nucl-th].

[53] M. Martini and M. Ericson, Phys. Rev. C 90, 025501 (2014)

[54] A. Meucci and C. Giusti, Phys. Rev. D 91, 093004 (2015).

[55] M. V. Ivanov, G. D. Megias, R. Gonzalez-Jimenez, O. Moreno, M. B. Barbaro, J. A. Caballero, and T. W. Donnelly, J. Phys. G 43, 045101 (2016).

[56] M. Martini, M. Ericson, and G. Chanfray, Phys. Rev. D 85, 093012 (2012).

[57] M. Martini, M. Ericson, and G. Chanfray, Phys. Rev. D 87, 013009 (2013).

[58] J. Nieves, F. Sanchez, I. R. Simo, and M. J. Vicente Vacas, Phys. Rev. D 85, 113008 (2012).

[59] O. Lalakulich, U. Mosel, and K. Gallmeister, Phys. Rev. C 86, 054606 (2012).

[60] T. Leitner and U. Mosel, Phys. Rev. C 81, 064614 (2010). 